\title{
IRBBO for Gain Maximization of Fifteen-Element Yagi-Uda Antenna
}

\author{
Gagan Sachdeva \\ Rayat Bahra Group of Institutes \\ Mohali Campus, Punjab [INDIA]
}

\author{
Dilpal Singh \\ UIET, Panjab University \\ Chandigarh [INDIA]
}

\begin{abstract}
Biogeography is the study of distribution of biological species, over space and time, among random habitats. Recently introduced Biogeography Based Optimization (BBO) is a technique, where solutions of the problem are termed as habitats. Feature, i.e., Suitability Index Variable (SIV), sharing among various habitats is made to occur with migration operator where as exploration of new SIVs is done with mutation operator. Yagi-Uda antenna is a widely used antenna design due to various useful properties of high gain, low cost and ease of construction. Immigration Refusal BBO (IRBBO) is a $\mathrm{BBO}$ variant introduced with the objective of improved performance and faster convergence. Designing a Yagi-Uda antenna involves determination of element lengths and spacings between them to get desired radiation characteristics. The gain of Yagi-Uda antenna is difficult to optimize as there is no analytical formula to determine gain directly, it makes relationship between antenna parameters and its characteristics highly complex and non-linear. In this paper, fifteen-element Yagi-Uda antenna is optimized for maximum gain using IRBBO. The obtained results are compared with Standard BBO, Bi-Swarm optimization, Ellipsoid Algorithm and Genetic Algorithm (GA). IRBBO shows better results among other compared optimization techniques for Yagi-Uda antenna design problem.
\end{abstract}

\section{Keywords:}

Yagi-Uda Antenna, Biogeography Based Optimization, Antenna Gain, Genetic Algorithm, Bi-Swarm Optimization, Ellipsoid Algorithm

\section{INTRODUCTION}

Antenna is an electrical device which converts electric signal into free space radiations and vice-versa. The various radiation characteristics that affect the design of an antenna are gain, impedance, bandwidth, frequency of operation, Side Lobe Level (SLL) etc. Yagi-Uda antenna is a widely used directional antenna design due to various desirable features, i.e., high forward gain, low cost and ease of construction. It is basically a parasitic linear array of parallel dipoles, one of which is energized directly by transmission line while the others act as parasitic radiators whose currents are induced by mutual coupling.

Yagi-Uda antenna was invented in 1926 by H. Yagi and S. Uda at Tohoku University [29] in Japan, however, published in English in 1928 [33]. The main objective, in design of Yagi-Uda antenna, is to find an optimum structure that meet certain radiation criteria like gain, impedance, SLL and beamwidth. However, due to its parasitic elements, it is extremely difficult to obtain an optimum design of Yagi-Uda antenna. Since its inception, Yagi- Uda antenna has been optimized several times for gain, impedance, SLL and bandwidth using different optimization techniques based on traditional mathematical approaches [23, 5, 9, 25, 8, 7, 10] and Artificial Intelligence (AI) techniques [16, 32, 31, 4, 18, 28, 27]. In 1949, Fishenden and Wiblin [15] proposed an approximate design of Yagi aerials for maximum gain, however, the approach was based on approximations. In 1959, Ehrenspeck and Poehler proposed a manual approach to maximize the gain of the antenna by varying various lengths and spacings of its elements [14].

Later on, with the availability of high performance computing, it became possible to optimize antennas numerically. Bojsen et al. in [5] proposed an optimization technique to find the maximum gain of Yagi-Uda antenna arrays with equal and unequal spacings between adjacent elements. Cheng et al. in [8] and [7] have used optimum spacings and lengths to optimize the gain of a Yagi-Uda antenna. In [10], Cheng has proposed optimum design of YagiUda antenna where antenna gain function is highly non-linear. The performance of these gradient based techniques depends on choice of initial solution.

In 1975, John Holland introduced Genetic Algorithms (GAs) as a stochastic, swarm based AI technique, inspired from natural evolution of species, to optimize arbitrary systems for certain cost function. Since then many researchers have used GAs to optimize Yagi-Uda antenna designs for gain, impedance and bandwidth separately [1, 16, 11] and collectively [32, 30, 17]. Jones et al. in [16] have used GA to optimize Yagi-Uda antenna for various radiation characteristics and compared the result with steepest gradient method. Baskar et al. in [4], have used Comprehensive Learning Particle Swarm Optimization (CLPSO) to optimize Yagi-Uda antenna and obtained better results than other optimization techniques. In [18], Li has optimized Yagi-Uda antenna using Differential Evolution (DE) and illustrated the capabilities of the proposed method with several Yagi-Uda antenna designs. In [28], Singh et al. have analyzed another useful, stochastic global search and optimization technique known as Simulated Annealing (SA) for the optimization of Yagi-Uda antenna. In 2008, Dan Simon introduced a new optimization technique based on science of biogeography, in which information sharing among various habitats, i.e., potential solutions, is obtained via migration operator and exploration of new features is done with mutation operator [26]. In [13], Du et al. have proposed the concept of immigration refusal in BBO aiming at improved performance. Singh et al. have presented BBO as a better optimization technique for Yagi-Uda antenna designs, as compared to other optimization techniques in [27]. In [19], Li has proposed the Bi-Swarm optimization technique to optimize the Yagi-Uda antenna and produced better result than GA, Particle Swarm Optimization (PSO) and Computer Intelligence (CI) techniques. In 2011, Amaral et al. has applied Ellipsoid algorithm to optimization of Yagi-Uda antenna for gain maximiza- 
tion [2]. Li et al. in |20| have used Invasive Weed Optimization (IWO) technique to optimize a six element Yagi-Uda antenna for maximum directivity.

In this paper, fifteen-element Yagi-Uda antenna has been optimized for gain maximization using IRBBO and results are compared with other optimization techniques. A method of moments based freeware programme, Numerical Electromagnetics Code 2 (NEC2), is used to evaluate the antenna designs for gain.

After this brief introduction, the paper is structured as follows: In Section 2, Yagi-Uda antenna is briefly discussed. Section 3 is dedicated to biogeography terminology, BBO technique and IRBBO. In Section 4, the design problem of fifteen-element Yagi-Uda antenna for gain maximization is presented and results obtained with IRBBO are compared with other optimization techniques. Finally, paper is concluded in Section 5.

\section{ANTENNA DESIGN PARAMETERS}

Yagi-Uda antenna is basically made of three types of elements: (a) Reflector (b) Feeder and (c) Directors. Reflector is longest of all elements and blocks radiations in one direction. Feeder or driven element is fed with the signal to be transmitted, directly from transmission line. Directors are usually more than one in number and are responsible for unidirectional radiations. Normally, there is no limit on number of directors, however, as the number of directors are increased beyond a certain limit there is a reduction in the induced current in the most extreme elements. Figure 1 presents a basic Yagi-Uda antenna design where all elements are placed along $y$-axis and parallel to $x$-axis. Middle segment of the reflector is placed at origin and signal to be transmitted is fed to the middle segment of the feeder element. An incoming field induces resonant currents on all the antenna elements which causes parasitic (reflector and directors) elements to re-radiate signals. These re-radiated fields are then picked up by the feeder element, that makes total current induced in the feeder equivalent to combination of the direct field input and the reradiated contributions from the director and reflector elements.

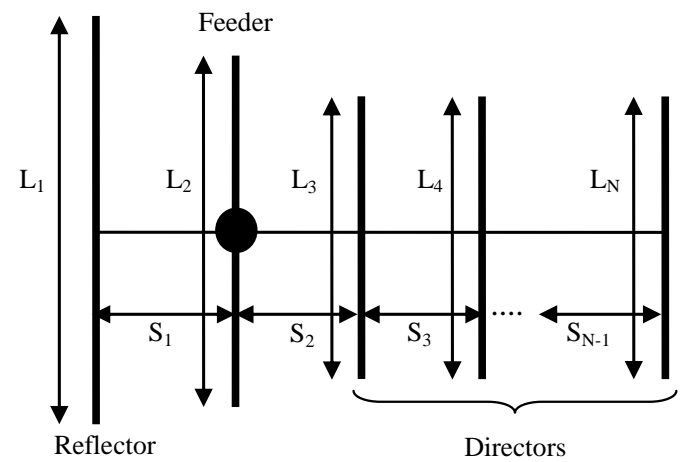

Fig. 1. Basic Yagi-Uda Antenna Design

Element lengths and spacings between them are the variables/parameters which need to be determined for optimum design of Yagi-Uda antenna. An antenna with $N$ elements requires $2 N-1$ parameters, i.e., $N$ wire lengths and $N-1$ spacings, to be determined. These $2 N-1$ parameters, collectively, are represented as an integer vector referred as a habitat in $\mathrm{BBO}$ given as (1).

$$
H=\left[L_{1}, L_{2}, \ldots, L_{N}, S_{1}, S_{2}, \ldots, S_{N-1}\right]
$$

where $L_{N}$ are the lengths and $S_{N-1}$ are the spacings between antenna elements.

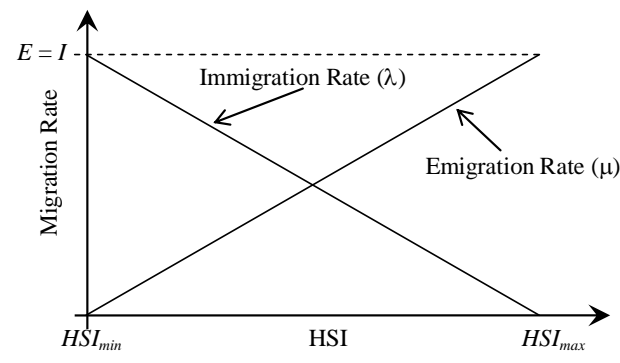

Fig. 2. Migration Curves

\section{BIOGEOGRAPHY BASED OPTIMIZATION}

Biogeography Based Optimization is a population based global optimization technique based on the science of biogeography, i.e., study of the distribution of animals and plants among different habitats over time and space. BBO results presented by researches, to optimize Yagi-Uda antenna, are better than other optimization techniques like PSO, GAs, SA, DE etc. [16, 30, 4, 22].

Initially, biogeography was studied by Alfred Wallace [3] and Charles Darwin [12] mainly as descriptive study. However, in 1967, the work carried out by MacAurthur and Wilson [21] changed this perception by introducing a mathematical model for biogeography which made it possible to predict the number of species in a habitat. Mathematical models of biogeography describe the migration, speciation and extinction of species in various habitats.

A habitat or island is an ecological area inhabited by a particular animal species which is geographically isolated from other habitats. Each habitat is characterized by its Habitat Suitability Index (HSI). Habitats which are well suited as living places for biological species are referred to have high HSI value. HSI is analogues to fitness in other Evolutionary Algorithms whose value is a function of many features of the habitat such as rainfall, diversity of vegetation, diversity of topographic features, land area, and temperature etc. The features/variables that characterize habitability are known as Suitability Index Variables (SIVs). In other words, HSI is dependent variable whereas SIVs are independent variables.

The habitats with high HSI have large probability of emigration (hence high emigration rate, $\mu$ ) simply due to large number of species they host and small probability of immigration (low immigration rate, $\lambda$ ) as they are already saturated with species. Immigration can be defined as the arrival of new species into a habitat, while emigration is the process of leaving one's native habitat. Similarly, habitats with low HSI tend to have low emigration rate, $\mu$, due to sparse population, however, they will have high immigration rate, $\lambda$. Suitability of habitats having low HSI value is likely to increase with more number of species arriving from habitats having high HSI as suitability of a habitat depends upon its biological diversity. For sake of simplicity, it is safe to assume a linear relationship between HSI (or population) and immigration and emigration rates. Also maximum emigration and immigration rates are assumed equal, i.e., $E=I$, as shown graphically in Figure 2.

For $k$-th habitat, values of emigration rate, $\mu_{k}$, and immigration rate, $\lambda_{k}$, are given by (2) and (3).

$$
\mu_{k}=E \cdot \frac{H S I_{k}}{H S I_{\max }-H S I_{\min }}
$$

$$
\lambda_{k}=I \cdot\left(1-\frac{H S I_{k}}{H S I_{\max }-H S I_{\min }}\right)
$$


Good solutions (habitats with high HSI) are more resistant to change than poor solutions (habitats with low HSI) whereas poor solutions are more dynamic in nature and accept a lot of new features from good solutions. This addition of new features to low HSI solutions from high HSI solutions may raise the quality of those solutions.

In a global optimization problem with number of possible solutions, each habitat or a solution in a population of size $N P$ is represented by $M$-dimensional integer vector as $H=$ $\left[S I V_{1}, S I V_{2}, \ldots, S I V_{M}\right]$ where $M$ is the number of SIVs (features) to be evolved for optimal HSI. HSI is the fitness criteria that is determined by evaluating the cost/objective function, i.e., $H S I=f(H)$. BBO consists of mainly two mechanisms: (A) Migration and (B) Mutation, these are discussed in the following subsections.

\subsection{Migration}

Migration is a probabilistic operator that improves HSI of poor habitats by sharing information from good habitats. During Migration, immigrating habitat, $I m H b t$, use its immigration rate, $\lambda$, given by (3), to probabilistically decide whether to immigrate or not. In case immigration is selected, then the emigrating habitat, $E m H b t$, is found probabilistically based on emigration rate, $\mu$, given by (2). The process of migration is completed by copying values of SIVs from EmHbt to ImHbt at random chosen sites. $\mathrm{BBO}$ employing above mentioned migration scheme is termed as the Standard BBO.

Different migration variants are proposed to increase the diversity in the population, with objective of improved performance of BBO algorithm. IRBBO is one of them and has shown impressive performance as compared to other $\mathrm{BBO}$ variants [13]. IRBBO is explained in following subsection. The pseudo codes for Standard BBO and IRBBO are depicted in Algorithm 1.

3.1.1 Immigration Refusal BBO (IRBBO). In BBO, a habitat with high emigration rate have high probability of emigration to other habitats and low probability of immigration from other habitats. However, the low immigration probability does not mean that immigration will never happen. Once in a while, a highly fit solution may receive solution features from a low-fit solution that may degrade its fitness. In such cases, immigration is refused to prevent degradation of fitness, i.e., HSI values of highly fit habitats. This BBO variant with conditional migration is termed as IRBBO and gives good performance with testbed of benchmark functions [13].

$\overline{\text { Algorithm } 1 \text { Pseudo Code of Migration for Standard BBO and }}$
IRBBO

Select ImHbt with probability based on $\lambda$

If ImHbt is selected then

Select EmHbt with probability based on $\mu$

If $E m H b t$ is selected then

Randomly select a SIV $(s)$ from $E m H b t$

switch(Option)

case 1: Standard

apply migration

case2: Immigration Refusal

if $($ fitness $(I m H b t)>$ fitness $(E m H b t))$

endif

apply migration

end switch

endif

endif

\subsection{Mutation}

Mutation is another probabilistic operator that alters the values of randomly selected SIVs of some habitats that are intended for exploration of search space for better solutions by increasing the biological diversity in the population. Here, higher mutation rates are investigated on habitats those are, probabilistically, participating less in migration process. Elitism approach is generally used along with mutation to preserve features of the best habitat. The mutation rate, $m$ Rate, for $k$-th habitat is calculated as (4)

$$
\text { mate }_{k}=C \times \min \left(\mu_{k}, \lambda_{k}\right)
$$

where $\mu_{k}$ and $\lambda_{k}$ are emigration and immigration rates, respectively, given by (2) and (3) corresponding to $H S I_{k}$. Here $C$ is a scaling constant and its value is equal to 1 . The pseudo code of mutation operator is given in Algorithm 2.

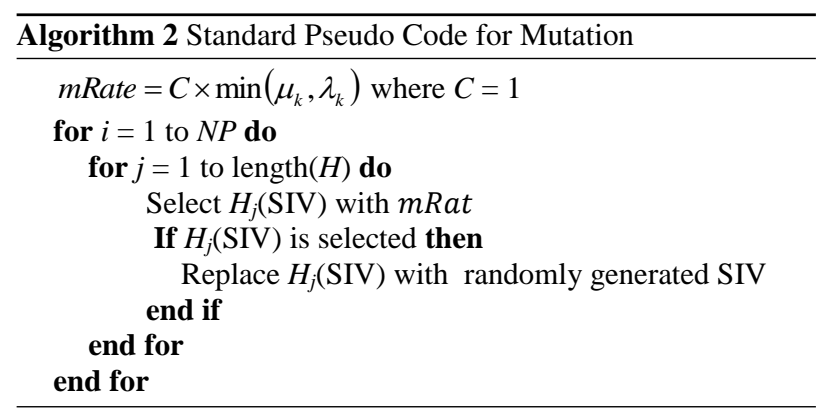

\section{SIMULATION RESULTS AND DISCUSSIONS}

Fifteen-wire Yagi-Uda antenna is optimized for maximum gain using IRBBO. To present a fair analysis, design is optimized with 30 habitats using 100 iterations. The $\mathrm{C}++$ programming environment is used for development of optimization algorithm, whereas, a method of moments based software named as $\mathrm{Nu}$ merical Electromagnetics Code 2 (NEC2) [6] is used for determination of required antenna characteristic, i.e., gain. Each potential solution in IRBBO is encoded as an integer vector with 29 SIVs as given by (1). The search spaces for the search of optimum values of wire lengths and wire spacings are $0.30 \lambda-0.50 \lambda$ and $0.10 \lambda-0.50 \lambda$, respectively. Cross sectional radius and segment sizes of all elements are kept constant, i.e., $0.003397 \lambda$ and $0.1 \lambda$ respectively, where $\lambda$ is the wavelength corresponding to frequency of operation, i.e., $300 \mathrm{MHz}$. The scaling constant C, the maximum migration rates $E$ and $I$, are set equal to 1 . The corresponding lengths and spacings obtained during optimization of Yagi-Uda antenna with IRBBO are tabulated in Table 1 along with other optimization techniques from published work. It can be seen from the Table 1 that gain of $18.44 \mathrm{dBi}$ obtained with IRBBO is more than obtained by Standard BBO [24], Biswarm optimization technique [19], Ellipsoid algorithm [2] and GA [16]. To the best of literature available, gain obtained by IRBBO, i.e., $18.44 \mathrm{dBi}$ is the highest gain that is obtained from a fifteen-element Yagi-Uda antenna yet.

\section{CONCLUSIONS AND FUTURE SCOPE}

In this paper, IRBBO algorithm is applied to optimize fifteenelement Yagi-Uda antenna designs for gain maximization. As per observations, the gain obtained with IRBBO is higher as compared to other optimization techniques including Standard BBO. Results show that IRBBO is a robust optimization technique for optimizing Yagi-Uda antenna. Investigation of Yagi-Uda antenna designs with different migration and mutation variants for better convergence performance is next on our agenda. 
Table 1. Results of Gain Optimized Fifteen-Element Yagi-Uda Antenna Designs

\begin{tabular}{|c|c|c|c|c|c|c|c|c|c|c|}
\hline & \multicolumn{2}{|c|}{ IRBBO } & \multicolumn{2}{|c|}{ Standard BBO [24] } & \multicolumn{2}{|c|}{ Bi-Swarm [19] } & \multicolumn{2}{|c|}{ Ellipsoid [2] } & \multicolumn{2}{|c|}{ GA [16] } \\
\hline Element & Length & Spacing & Length & Spacing & Length & Spacing & Length & Spacing & Length & Spacing \\
\hline $1(\lambda)$ & 0.4840 & - & 0.4808 & - & 0.4855 & - & 0.4561 & - & 0.474 & - \\
\hline $2(\lambda)$ & 0.4647 & 0.2818 & 0.4581 & 0.2690 & 0.4557 & 0.2397 & 0.4510 & 0.3788 & 0.486 & 0.356 \\
\hline $3(\lambda)$ & 0.4458 & 0.1856 & 0.4441 & 0.1963 & 0.4399 & 0.2810 & 0.4490 & 0.3921 & 0.452 & 0.144 \\
\hline $4(\lambda)$ & 0.4294 & 0.3512 & 0.4274 & 0.3716 & 0.4311 & 0.3688 & 0.4430 & 0.4036 & 0.436 & 0.340 \\
\hline $5(\lambda)$ & 0.4172 & 0.4541 & 0.4154 & 0.4199 & 0.4259 & 0.3881 & 0.4375 & 0.4088 & 0.414 & 0.447 \\
\hline $6(\lambda)$ & 0.4126 & 0.4135 & 0.4061 & 0.4782 & 0.4215 & 0.3837 & 0.4445 & 0.4249 & 0.420 & 0.362 \\
\hline $7(\lambda)$ & 0.4065 & 0.4611 & 0.4067 & 0.4460 & 0.4043 & 0.4850 & 0.4473 & 0.4307 & 0.414 & 0.370 \\
\hline $8(\lambda)$ & 0.4051 & 0.4377 & 0.4029 & 0.4410 & 0.4054 & 0.4712 & 0.4431 & 0.4272 & 0.398 & 0.395 \\
\hline $9(\lambda)$ & 0.3995 & 0.4973 & 0.3970 & 0.4728 & 0.4033 & 0.4845 & 0.4373 & 0.4301 & 0.414 & 0.414 \\
\hline $10(\lambda)$ & 0.3998 & 0.4573 & 0.4017 & 0.4597 & 0.4094 & 0.4144 & 0.4386 & 0.4874 & 0.376 & 0.425 \\
\hline $11(\lambda)$ & 0.4008 & 0.4411 & 0.4013 & 0.4458 & 0.4028 & 0.4614 & 0.4229 & 0.3911 & 0.338 & 0.296 \\
\hline $12(\lambda)$ & 0.3963 & 0.4677 & 0.3991 & 0.4811 & 0.4074 & 0.4580 & 0.4213 & 0.4391 & 0.398 & 0.334 \\
\hline $13(\lambda)$ & 0.4008 & 0.4693 & 0.3991 & 0.4472 & 0.3936 & 0.5157 & 0.4385 & 0.3977 & 0.410 & 0.348 \\
\hline $14(\lambda)$ & 0.4032 & 0.4535 & 0.4015 & 0.4530 & 0.3955 & 0.4537 & 0.4867 & 0.4057 & 0.408 & 0.392 \\
\hline $15(\lambda)$ & 0.4127 & 0.4245 & 0.4146 & 0.4579 & 0.4142 & 0.4317 & 0.4293 & 0.2263 & 0.398 & 0.450 \\
\hline Gain (dBi) & \multicolumn{2}{|c|}{18.44} & \multicolumn{2}{|c|}{18.41} & \multicolumn{2}{|c|}{18.31} & \multicolumn{2}{|c|}{17.48} & \multicolumn{2}{|c|}{17.07} \\
\hline
\end{tabular}

\section{REFERENCES}

[1] E.E. Altshuler and D.S. Linden. Wire-antenna Designs using Genetic Algorithms. Antennas and Propagation Magazine, IEEE, 39(2):33-43, 1997.

[2] A.N. Amaral, U.C. Resende, and E.N. Gonçalves. Yagi-uda antenna optimization by elipsoid algorithm. pages 503 506, 2011.

[3] A.Wallace. The Geographical Distribution of Animals. Boston, MA: Adamant Media Corporation, Two:232-237, 2005.

[4] S. Baskar, A. Alphones, P N Suganthan, and J J Liang. Design of Yagi-Uda Antennas using Comprehensive Learning Particle Swarm Optimisation. IEEE, 152(5):340-346, 2005.

[5] JH Bojsen, H. Schjaer-Jacobsen, E. Nilsson, and J. Bach Andersen. Maximum Gain of Yagi-Uda Arrays. Electronics Letters, 7(18):531-532, 1971.

[6] G. J. Burke and A. J. Poggio. Numerical Electromagnetics Code (NEC) method of moments. NOSC Tech. DocLawrence Livermore National Laboratory, Livermore, Calif, USA, 116:1-131, 1981.

[7] C. Chen and D. Cheng. Optimum Element Lengths for Yagi-Uda Arrays. IEEE Transactions on Antennas and Propagation, 23(1):8-15, 1975.

[8] D. Cheng and C. Chen. Optimum Element Spacings for Yagi-Uda Arrays. IEEE Transactions on Antennas and Propagation, 21(5):615-623, 1973.

[9] D. K. Cheng. Optimization Techniques for Antenna Arrays. Proceedings of the IEEE, 59(12):1664-1674, 1971.

[10] D. K. Cheng. Gain Optimization for Yagi-Uda Arrays. Antennas and Propagation Magazine, IEEE, 33(3):42-46, 1991.

[11] D. Correia, A. J. M. Soares, and M. A. B. Terada. Optimization of gain, impedance and bandwidth in Yagi-Uda Antennas using Genetic Algorithm. IEEE, 1:41-44, 1999.

[12] C. Darwin. The Orign of Species. New York : gramercy, Two:398-403, 1995.

[13] D. Du, D. Simon, and M. Ergezer. Biogeography-based Optimization Combined with Evolutionary Strategy and Immigration Refusal. IEEE, 1:997-1002, 2009.
[14] H. Ehrenspeck and H. Poehler. A New Method for Obtaining Maximum Gain from Yagi Antennas. IRE Transactions on Antennas and Propagation, 7(4):379-386, 1959.

[15] R. M. Fishenden and E. R. Wiblin. Design of Yagi Aerials. Proceedings of the IEE-Part III: Radio and Communication Engineering, 96(39):5, 1949.

[16] E. A. Jones and W. T. Joines. Design of Yagi-Uda Antennas using Genetic Algorithms. IEEE Transactions on Antennas and Propagation, , 45(9):1386-1392, 1997.

[17] Y. Kuwahara. Multiobjective Optimization Design of YagiUda Antenna. IEEE Transactions on Antennas and Propagation, 53(6):1984-1992, 2005.

[18] J. Y. Li. Optimizing Design of Antenna using Differential Evolution. IEEE, 1:1-4, 2007.

[19] J.Y. Li. A bi-swarm optimizing strategy and its application of antenna design. Journal of Electromagnetic Waves and Applications, 23(14-15):1877-1886, 2009.

[20] Y. Li, F. Yang, J. OuYang, and H. Zhou. Yagi-uda antenna optimization based on invasive weed optimization method. Electromagnetics, 31(8):571-577, 2011.

[21] R.H. MacArthur and E.O. Wilson. The Theory of Island Biogeography. Princeton Univ Pr, 1967.

[22] M. Rattan, M. S. Patterh, and B. S. Sohi. Optimization of Yagi-Uda Antenna using Simulated Annealing. Journal of Electromagnetic Waves and Applications, 22, 2(3):291299, 2008.

[23] D. G. Reid. The Gain of an Idealized Yagi Array. Journal of the Institution of Electrical Engineers-Part IIIA: Radiolocation, 93(3):564-566, 1946.

[24] G. Sachdeva, D. Singh, and S. Singh. Gain Optimization of 15-Element Yagi-Uda Antenna using BBO. In proceeding of IEEE, 2013.

[25] L. C. Shen. Directivity and Bandwidth of Single-band and Double-band Yagi Arrays. IEEE Transactions on Antennas and Propagation, 20(6):778-780, 1972.

[26] D. Simon. Biogeography-based Optimization. IEEE Transactions on Evolutionary Computation,, 12(6):702-713, 2008.

[27] U. Singh, H. Kumar, and T. S. Kamal. Design of Yagi-Uda Antenna Using Biogeography Based Optimization. IEEE Transactions on Antennas and Propagation, , 58(10):33753379,2010 . 
[28] U. Singh, M. Rattan, N. Singh, and M. S. Patterh. Design of a Yagi-Uda Antenna by Simulated Annealing for Gain, Impedance and FBR. IEEE, 1:974-979, 2007.

[29] Shintaro Uda and Yasuto Mushiake. Yagi-Uda Antenna. Maruzen Company, Ltd, 1954.

[30] N. V. Venkatarayalu and T. Ray. Single and Multi-Objective Design of Yagi-Uda Antennas using Computational Intelligence. IEEE, 2:1237-1242, 2003.

[31] N.V. Venkatarayalu and T. Ray. Optimum Design of YagiUda Antennas Using Computational Intelligence. IEEE
Transactions on Antennas and Propagation, 52(7):18111818, 2004.

[32] H. J. Wang, K. F. Man, C. H. Chan, and K. M. Luk. Optimization of Yagi array by Hierarchical Genetic Algorithms. IEEE, 1:91-94, 2003.

[33] H. Yagi. Beam Transmission of Ultra Short Waves. Proceedings of the Institute of Radio Engineers, 16(6):715740, 1928. 